\title{
Wide complex tachycardia - diagnostic and therapeutic challenges
}

\author{
Claudia Elena Bezim ${ }^{1}$, Carina Gabriela Ureche*,1, Radu Andy Sascău ${ }^{1,2}$, Mihaela \\ Grecu $^{4}$, Grigore Tinică ${ }^{2,3}$, Cătălina Arsenescu Georgescu ${ }^{1,2}$ Cristian Stătescu ${ }^{2,4}$
}

1Department of Cardiology, "Prof. Dr. George I. M. Georgescu" Institute of Cardiovascular Diseases, lasi, Romania. ${ }^{2 " G r i g o r e ~ T . ~ P o p a " ~ U n i v e r s i t y ~ o f ~ M e d i c i n e ~ a n d ~ P h a r m a c y, ~ l a s i, ~ R o m a n i a . ~}{ }^{3}$ Department of Cardiovascular Surgery, "Prof. Dr. George I. M. Georgescu" Institute of Cardiovascular Diseases, lasi, Romania. ${ }^{4}$ Department of Electrophysiology, "Prof. Dr. George I. M. Georgescu" Institute of Cardiovascular Diseases, lasi, Romania

\begin{abstract}
Dilated cardiomyopathy is associated with ventricular arrhythmias and with an increased risk of sudden cardiac death. In these cases, wide complex tachyarrhythmias are frequently a diagnostic challenge. The efficiency of antiarrhythmic drug therapy is limited and often the implantation of a cardiac defibrillator is required. We hereby present the case of a 68 year old male patient known with dilated cardiomyopathy, who experienced a syncopal episode as the clinical expression of a wide complex tachycardia. The recorded electrocardiogram identified ventricular tachycardia originated in the right ventricle or in the interventricular septum. The electrocardiograms recorded before and after the syncopal episode showed a major left bundlebranch block and a first degree atrioventricular block. 24-hour Holter ECG monitoring detected ventricular tachycardia originating in the left ventricle. The etiology of the dilated cardiomyopathy, arrhythmias and conduction abnormalities could not be identified. During the electrophysiological study, no sustained supraventricular or ventricular tachyarrhythmias were triggered. The pharmacological treatment has been optimized and a cardiac defibrillator was implanted. The particularity of this case consists in the presence of multiple rhythm disturbances originating both in the left and right ventricle, in a patient with idiopathic dilated cardiomyopathy and conduction disturbances at several levels.
\end{abstract}

Keywords: idiopathic dilated cardiomyopathy, wide complex tachycardia

\section{Introduction}

Myocardial diseases that evolve with progressive heart dilation and systolic dysfunction are frequently encountered in the clinical practice. They have different causes from idiopathic dilated cardiomyopathy-DCM to secondary DCM due to coronary artery

Received: February 2017; Accepted after review: June 2017; Published: June 2017.

*Corresponding author: Carina Gabriela Ureche, MD, Department of Cardiology, "Prof. Dr. George I. M. Georgescu" Institute of Cardiovascular Diseases, Bulevardul Carol I, Nr. 50, lași 700503, lasi, Romania. E-mail: carina.ureche@yahoo.com disease, alcohol consumption, arterial hypertension or advanced valvular heart disease. DCM is often associated with cardiac arrhythmias, usually originating in the left ventricle. Wide complex tachyarrhythmias can be a real diagnostic challenge [1].

In most cases, the electrophysiological study induces ventricular rhythm disorders. Amiodarone therapy effectiveness is limited, thus the implantation of a cardiac defibrillator is often required [2].

\section{Case report}

We hereby present the case of a 68 yearold male, known with DCM of unknown 
etiology, who had a syncopal episode with no prodrome 86 days prior to the admission, from which he recovered spontaneously. The ECG recorded (Figure 1) suggests ventricular tachycardia originating in the right ventricle or in the interventricular septum. The patient was treated with synchronized direct current cardioversion and regained sinus rhythm. Subsequently, the patient was treated with amiodarone.

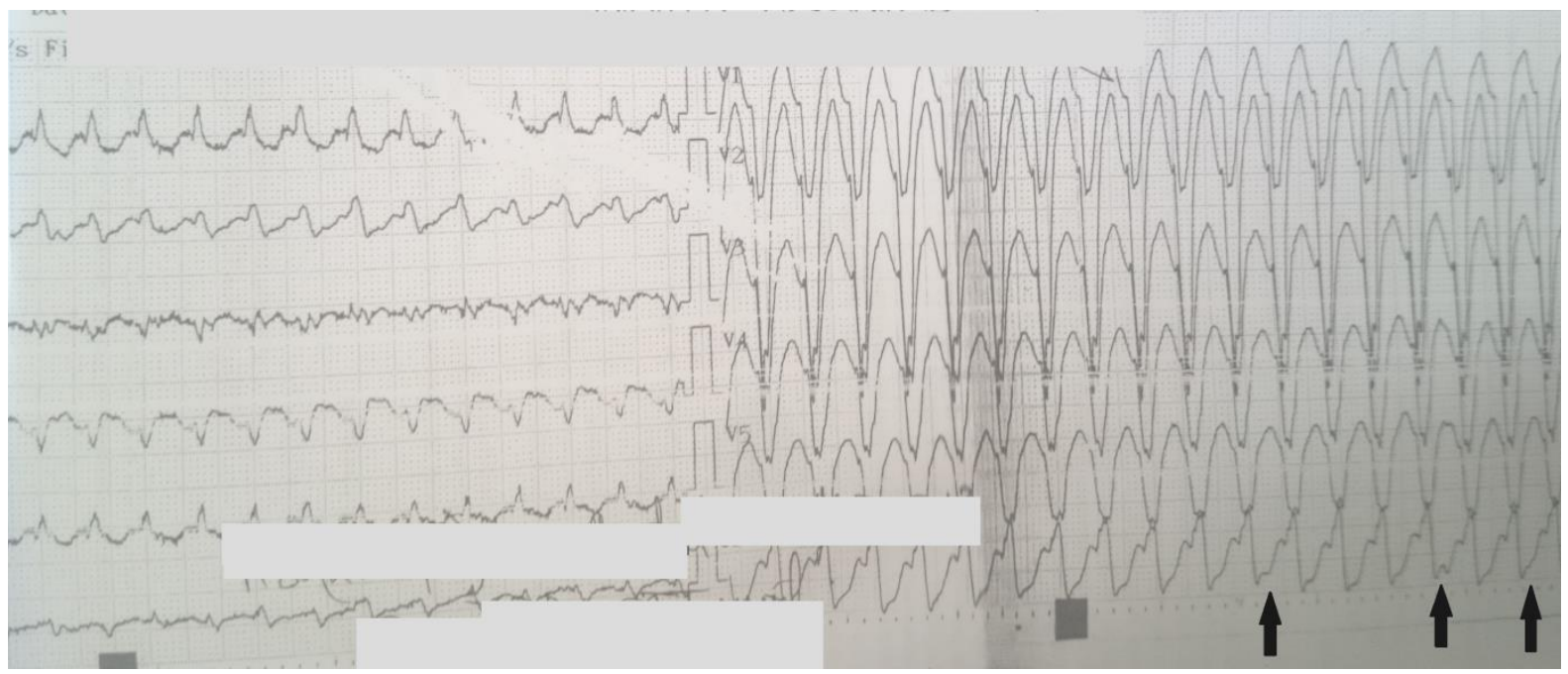

Fig. 1. Crisis ECG: Wide complex tachycardia, with LBBB aspect and left QRS axis, HR 200 bpm

Physical examination identified a systolic murmur, IV / VI, located at the apex, with left axillary irradiation. The ECG at admission is shown in Figure 2.

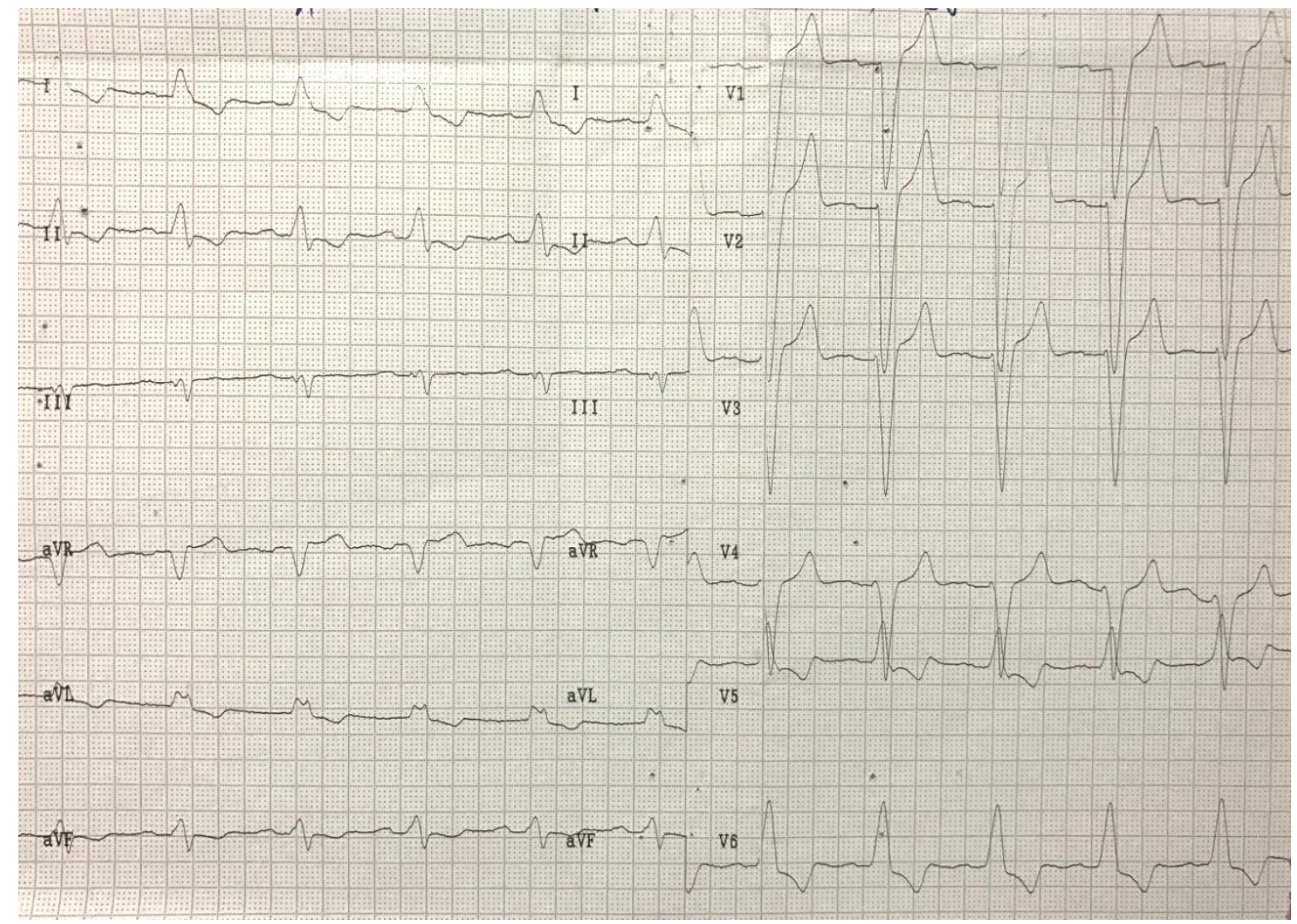

Fig. 2. $\mathrm{SR}, 75 \mathrm{bpm}, \mathrm{QRS}$ axis at 0 degrees, complete LBBB (QRS=160 ms), first degree $A V$ block $(P R$ interval $=$ $200 \mathrm{~ms}), \mathrm{QT}=520 \mathrm{~ms}, \mathrm{JT}=320 \mathrm{~ms}$ 
The patient had an impaired renal function $\left(\mathrm{Cr}=1.25 \mathrm{mg} / \mathrm{dl}, \mathrm{Cl} \mathrm{Cr}=57 \mathrm{ml} / \mathrm{min} / 1.73 \mathrm{~m}^{2}\right.$ MDRD formula) and a potassium level of 5 $\mathrm{mmol} / \mathrm{l}$.

In order to exclude myocardial ischemia as the cause of DCM and ventricular tachycardia, coronary computed tomography angiogram was performed, but no epicardial coronary lesions were found.

The patient denies alcohol consumption, a history of hypertension or diabetes (fasting glucose levels of $97 \mathrm{mg} / \mathrm{dl}$ ), hypothyroidism or recent viral infection. There were no heart diseases known in his family.

Electrophysiological study was performed, in order to assess the possibility of ablation, with both atrial and ventricular stimulation, without triggering any supraventricular or sustained ventricular tachyarrhythmias.
The transthoracic echocardiography revealed global dilation of the heart, predominantly left cavities $(\mathrm{LA}=54 \mathrm{~mm}$, LVEDD $=61 \mathrm{~mm}$, LVESD $=55 \mathrm{~mm}$, LVEDV $=$ $270 \mathrm{ml}$, LVESV $=210 \mathrm{ml}$ ), moderate mitral regurgitation (MR) secondary to left ventricular dilation. We identified a type III b Carpentier MR, through systolic restriction of cusps mobility. The echocardiography identified an impaired systolic function with an ejection fraction of $20 \%$, global hypokinesia and an abnormal septal movement due to LBBB.

The 24-hour Holter monitoring (Figure 3) identified a heart rate between 47 and 109 bpm, without significant pauses or atrial tachyarrhythmias. We mention that the patient was undergoing treatment with amiodarone upon registration, and the tachycardic episode was asymptomatic.

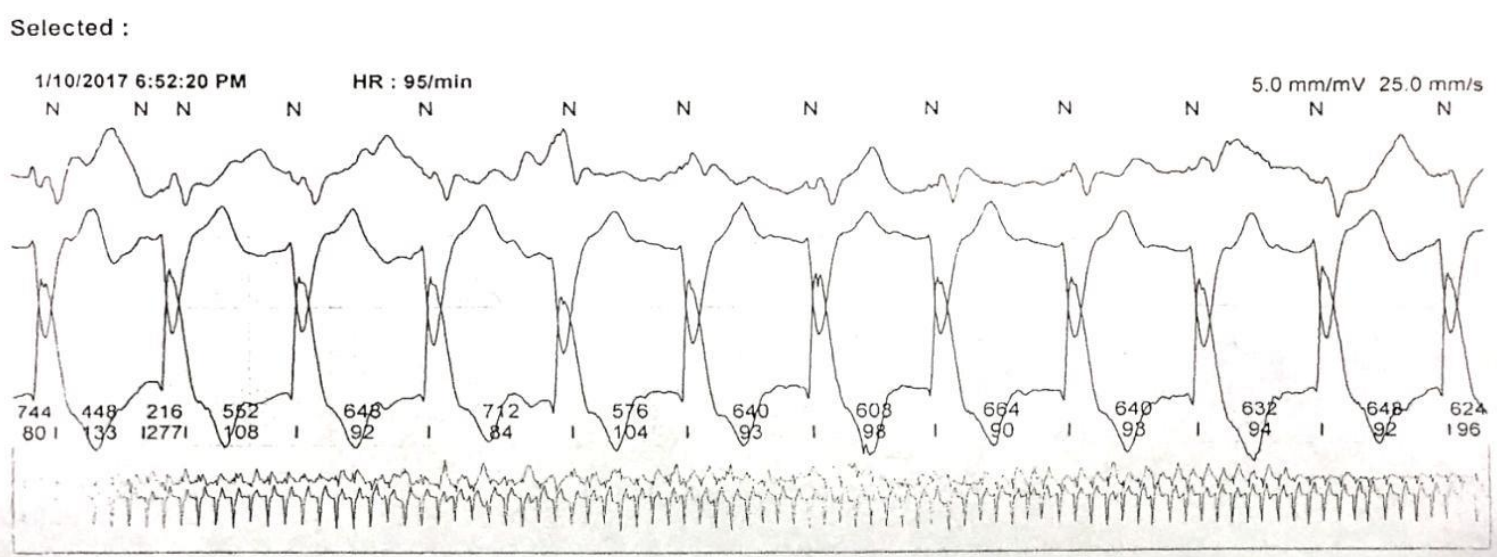

Comments :

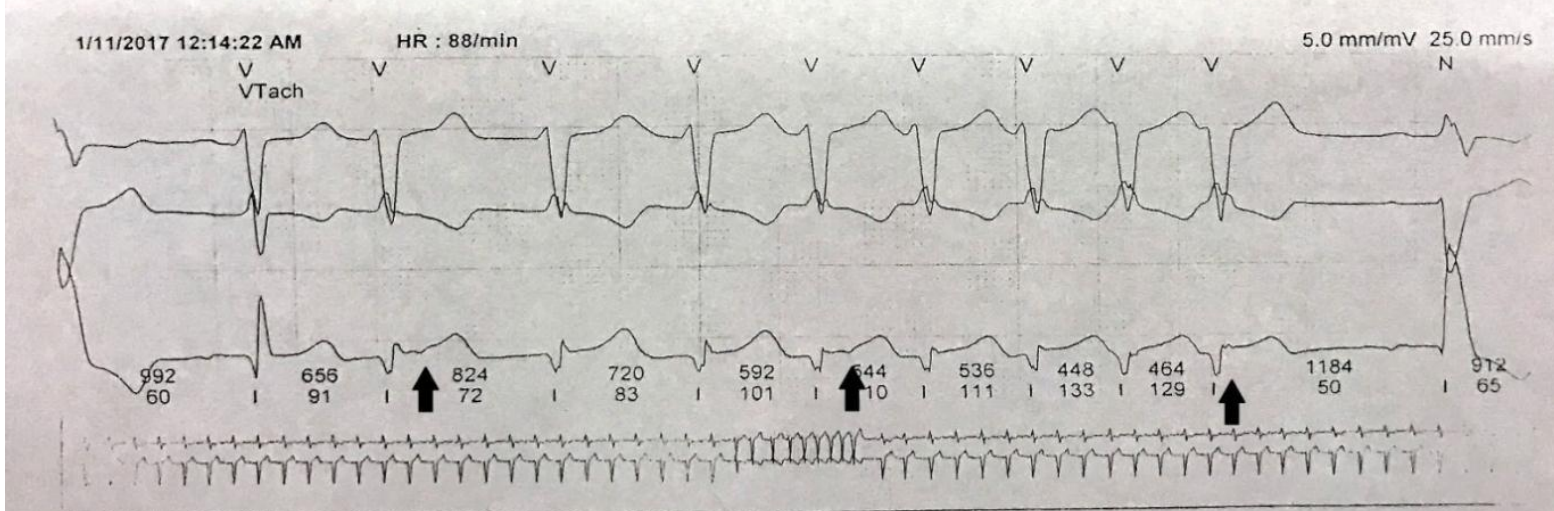

Fig. 3. Holter monitoring: Ventricular tachycardia with RR' morphology in V1 with R> R', 100 / min, with AV dissociation (arrows) 
Given the episode of ventricular tachycardia and that the patient's life expectancy exceeds one year, an implantable cardioverter defibrillator with DDD stimulation mode was implanted. The post-implant ECG shows ventricular stimulated complexes, with small, barely visible spikes (Figure 4).

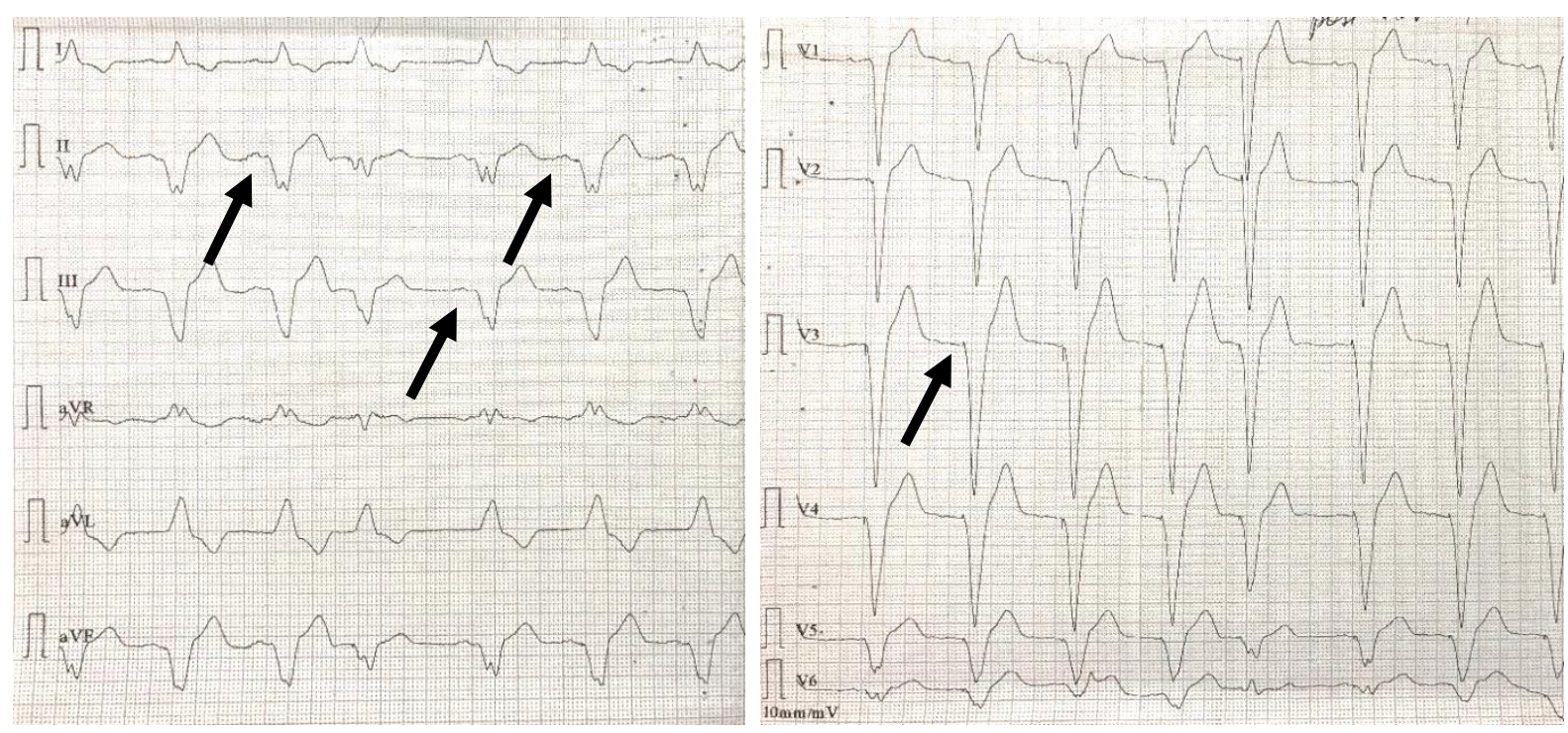

Fig. 4. Post-implant ECG, stimulated ventricular complexes (arrows).

Left: Limb leads; Right: Precordial leads

Considering that the patient had nonischemic cardiomyopathy with LVEF $20 \%$, was in sinus rhythm, with LBBB morphology wider than $150 \mathrm{~ms}$, resynchronization therapy was brought into discussion. The patient had NYHA class I heart failure symptoms, with no limitation in ordinary physical activity. At the moment of the admission there were no resynchronization devices available in the hospital. The patient could benefit from resynchronization therapy in a subsequent admission.

Pharmacological treatment has been optimized by introducing an angiotensinconverting enzyme (ACE) inhibitor, a betablocker, an aldosterone blocker, in order to reduce progression to heart failure and sudden cardiac death risk. The patient's serum creatinine increased with $0.8 \mathrm{mg} / \mathrm{dl}$, so the treatment with ACE inhibitor was suspended.

Treatment with amiodarone was continued for the prevention of ventricular arrhythmias and therefore internal electric shocks.

\section{Discussions}

The crisis electrocardiogram (Figure 1) is suitable for differential diagnosis between supraventricular tachycardia with aberrant conduction and ventricular tachycardia. In order to elucidate the electric diagnosis, the Brugada criteria apply [3]. Looking at the precordial leads, we see no negative / positive concordance: $r S$ present in V2-V4. Duration of the R-S in the chest leads is less than $100 \mathrm{~ms}$. We can observe atrioventricular dissociation, as $P$ waves that distort the QRS complexes (arrows, Figure 1) - a strong indicator of ventricular tachycardia. Therefore, it is safe to say that the rhythm disorder originates in the right ventricle or in the interventricular septum.

At the time of the electrocardiogram, the patient was not receiving any pro-arrhythmic medication and no serum electrolyte abnormalities which could be incriminated in the etiology of the rhythm disorder were found.

Holter monitoring identified ventricular tachycardia with RR' morphology in V1 with R> R', 100/min, with AV dissociation (Figure 3, arrows). The aspect of the QRS complex suggests a left ventricular origin [4]. The 
diagnosis is supported by the presence of various premature ventricular contractions with the same layout (Figure 5). In Figure 5, there is a $P$ wave but the $P R$ interval is shorter than usual, which means that the QRS complex is of ventricular origin.

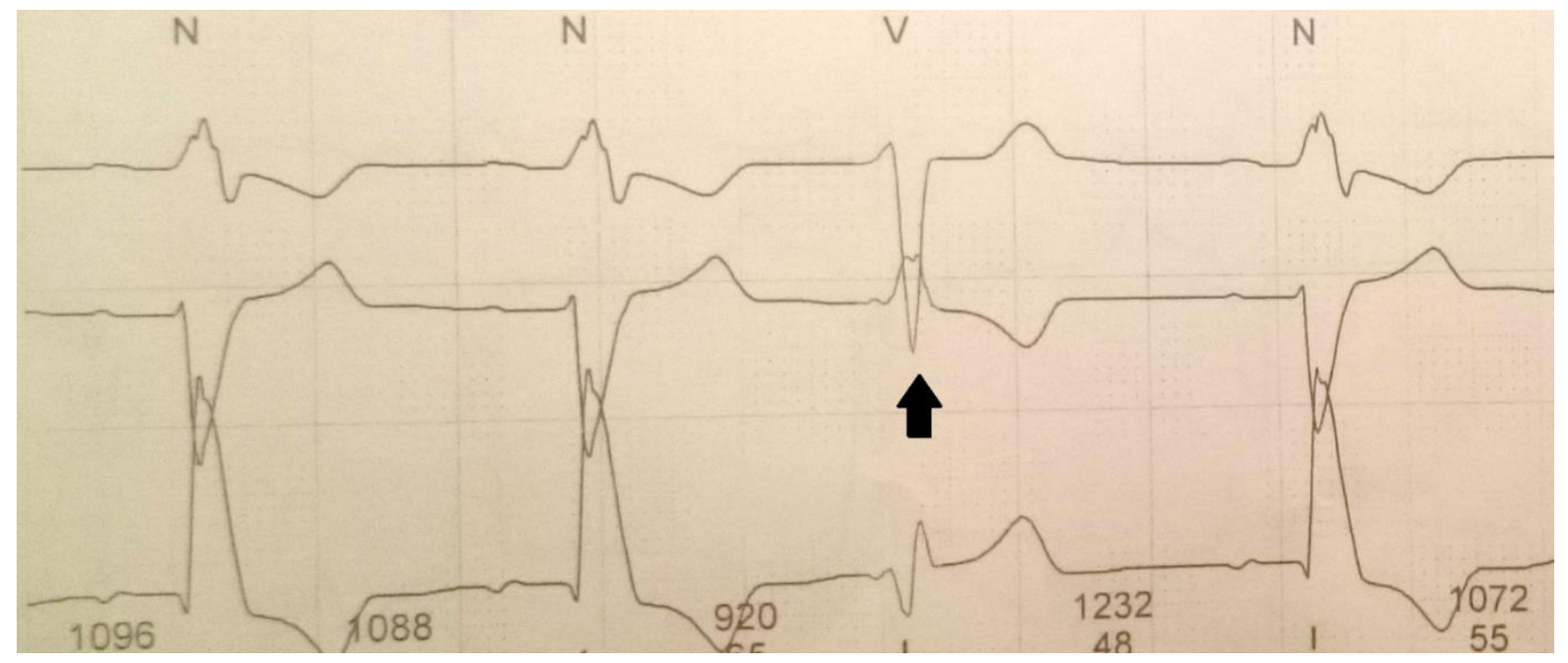

Fig. 5. Premature ventricular contraction

The lengthening of the PR interval may indicate an infrahisian conduction disorder located on the right bundle or a delay in the atrioventricular node [5]. The association between LBBB and first degree AV block represents a risk factor for developing a complete atrioventricular block. Given the incomplete trifascicular block and the syncopal episode, it is safe to assume that a possible cause of the syncope could be an intermittent complete AV block [5].

Increased duration of the QRS complex indicates a high degree of electrical interventricular asynchrony, which is correlated with mechanical asynchrony.

The patient was under treatment with amiodarone at the time of the electrophysiological study. We can assume that the antiarrhythmic treatment prevented the occurrence of tachyarrhythmia.

The indication for cardiac MRI was brought into discussion, [6] in order to identify an anatomical trigger substrate for arrhythmias and conduction disturbances, but the presence of metallic foreign objects in the left elbow and in the scapula prohibited the investigation.

It is difficult to assess the accuracy of measurement of the corrected QT interval in patients with LBBB and thus to monitor treatment with amiodarone. This results in the risk of developing iatrogenic arrhythmias when the QT interval increases excessively under treatment with amiodarone. A wide QRS complex leads to an increased QTc interval which is not relevant. In order to be more accurate, electrophysiologists suggest that a corrected JT (JTc) interval could be used in these patients. It is obtained through the Bazett formula: JTC $=$ JT/RR interval. JTC interval above $310 \mathrm{~ms}$ is considered abnormal. [7]. We calculated a JTc interval of $340 \mathrm{~ms}$ and amiodarone dose was decreased. Amiodarone discontinuation was considered, but the medical team decided to continue administration one more month, until the next follow-up.

Patient maintained sinus rhythm, although the cardiac cavities presented important dilation. There was no atrial fibrillation identified on Holter monitoring.

\section{Conclusions}

The patient was diagnosed with idiopathic dilated cardiomyopathy - normal coronary arteries, no alcohol consumption or cardiac toxic substances abuse, no history of infectious disease, no thyroid pathology, hypertension, diabetes or valve abnormalities. The patient suffers from chronic kidney 
disease, which limited the administration of ACE inhibitors.

The particularity of the case is represented by the fact that although the patient's heart was globally dilated and the ECG showed a LBBB and a first degree AV block, he maintained sinus rhythm. The patient presented an episode of sustained ventricular tachycardia, with its origin in the right ventricle or in the interventricular septum, with a heart rate of $200 / \mathrm{min}$, having syncope as the main clinical manifestation; nevertheless, nonsustained ventricular tachycardia originating in the left ventricle was identified on the 24 hour Holter monitoring [1], with RR' morphology in

\section{References}

1. Garmel GM. Wide Complex tachycardias: understanding this complex condition Part 2 Management, miscellaneous causes, and pitfalls. West J Emerg Med 2008; 9(2):97-103.

2. Poll D, Marchlinski F, Buxton A, Doherty J, Waxman $H$, Josephson $M$. Sustained ventricular tachycardia in patients with idiopathic dilated cardiomyopathy: electrophysiologic testing and lack of response to antiarrhythmic drug therapy. Circulation 1984; 70(3):451-456.

3. Olgin J, Zipes D. Specific Arrhythmias: Diagnosis and Treatment. In Braunwald's Heart Disease - A Textbook of Cardiovascular Medicine, $10^{\text {th }} \quad$ Edition. Philadelphia: Elsevier/Saunders. 2015; 776-780.

4. Burns E: Ventricular tachycardia - Life in the Fast lane ECG Library [http://lifeinthefastlane.com/ecg-
V1, R> R' 100/min, but completely asymptomatic.

In conclusion, the patient presents conduction abnormalities at different levels and arrhythmias which origin in both ventricles.

\section{Consent}

Written informed consent was obtained from the patient for publication of this case report and accompanying images. A copy of the written consent is available for review by the Editor-in-Chief of this journal.

\section{Conflict of interest}

Authors declare no conflicts of interest.

library/ventricular-tachycardia/ available at 29.01.2017].

5. Burns E: Trifascicular Block - Life in the Fastlane ECG Library. [http://lifeinthefastlane.com/ecglibrary/trifascicular-block/ available at 29.01.2017].

6. Priori $S$, Blomström-Lundqvist $C$, Mazzanti $A$, et al.; 2015 ESC Guidelines for the management of patients with ventricular arrhythmias and the prevention of sudden cardiac death: The task force for the management of patients with ventricular arrhythmias and the prevention of sudden cardiac death of the European Society of Cardiology (ESC). Eur Heart J 2015; 36 (41): 2804.

7. Tabatabaei $P$, Keikhavani $A$, Haghjoo $M$ et al. Assessment of QT and JT intervals in patients with left bundle branch block. Res Cardiovasc Med 2016; 5(2): e31528. 